\title{
Mechanochemical Ritter reaction: a rapid approach to functionalized amides at room temperature
}

\author{
Irena Dokli and Matija Gredičak*[a]
}

\begin{abstract}
A fast and efficient mechanochemical Ritter reaction between alcohols and nitriles under mild conditions is demonstrated. The reaction proceeds rapidly at room temperature in a solvent-free or low-solvent environment, utilizing a Brønsted acid catalyst. Its general application has been verified through a substrate screening investigation comprising a wide range of functionalized nitriles, as well as secondary and tertiary alcohols.
\end{abstract}

\section{Introduction}

The Ritter reaction is an organic reaction that allows formation of amides from a carbocation precursor (tertiary alcohol or substituted olefin) and a nitrile using a strong acid catalyst. ${ }^{[1]}$ Although the Ritter reaction found its application in drug, ${ }^{[2]}$ and natural product and natural product-like syntheses, ${ }^{[3]}$ the traditional use of stoichiometric amounts of strong corrosive acids at elevated temperatures limits its wider application in terms of functional group stability.

Various procedures comprising sub-stoichiometric amounts of mineral acids (usually sulfuric acid) have been reported. ${ }^{[4]}$ However, over the past decade, non-nucleophilic organic Brønsted acids have emerged as viable catalysts, mostly sulfonic acids ${ }^{[5]}$. The Ritter and Ritter-type reactions have also been successfully catalysed with organic ${ }^{[6]}$ and metal[ ${ }^{[7]}$ Lewis acids.

On the other hand, research attempts to perform the reaction under mild conditions, and thus making it more environmentally friendly, were met with limited success. Shorter reaction times were achieved utilizing $\mathrm{FeCl}_{3}{ }^{[7 \mathrm{c}]}$ and $\mathrm{Ca}(\mathrm{OTf})_{2}{ }^{[\mathrm{e}]}$ catalysts, though high temperatures were still required. A fast protocol at room temperature with an excess of sulfuric acid was established ${ }^{[8]}$ however, the reaction was substrate specific for tert-butyl acetate as a carbocation precursor.

In further pursue of convenient and efficient Ritter reaction, solvent-free procedures employing solid-supported catalysts ${ }^{[9]}$ and ionic liquids ${ }^{[10]}$ have been reported, but the main issue remains high reaction temperature. Recently, an environmentally benign solvent-free protocol at room temperature was published, however, the reaction time was substantially prolonged. ${ }^{[11}$ Neutral and mild Ritter reaction was achieved with gold(I) catalyst, yet the protocol was not suitable for solid nitriles, as nitrile was used as the solvent. ${ }^{[12]}$

In addition, abovementioned protocols mostly describe employment of non-functionalized nitriles as substrates, and provide a narrow substrate scope; functionalized amides are still

[a] Dr. I. Dokli, Dr. M. Gredičak

Division of Organic Chemistry and Biochemistry

Ruđer Bošković Institute

Bijenička cesta 54, 10000 Zagreb

E-mail: matija.gredicak@irb.hr

Supporting information for this article is given via a link at the end of the document. mostly prepared by standard acid chloride-amine couplings or by recently developed ortho-directed functionalizations, ${ }^{[13]}$ metalcatalysed amidations ${ }^{[14]}$ and metal-free carboxyamidations. ${ }^{[15]}$

In this paper, we report a general procedure for Brønsted acid catalysed mechanochemical Ritter reaction under mild conditions: room temperature, short reaction time, and a solventfree or low-solvent environment. The versatility of the protocol is veryfied through a wide substrate scope investigation, including functionalized nitriles, as well as secondary and tertiary alcohols.

Mechanochemistry has been recognized as one of the most successful modes of solvent-free synthesis. ${ }^{[16]}$ Mechanochemical reactions, usually performed in ball mills, are now present in all fields of chemistry, and their application in organic synthesis is increasing. ${ }^{[17]}$ Recently, it has been shown that conditions produced by a ball mill could be compared to those produced when performing the same reaction at elevated temperature in a solution, though the temperature in the vial remains virtually ambient. ${ }^{[18]}$ Hence, we reasoned that the activation energy of the Ritter reaction could be overcome during ball milling.

\section{Results and Discussion}

In order to test our hypothesis, we prepared a model reaction between benzonitrile and tert-butanol ${ }^{[19]}$ with Brønsted acid catalyst (Table 1).

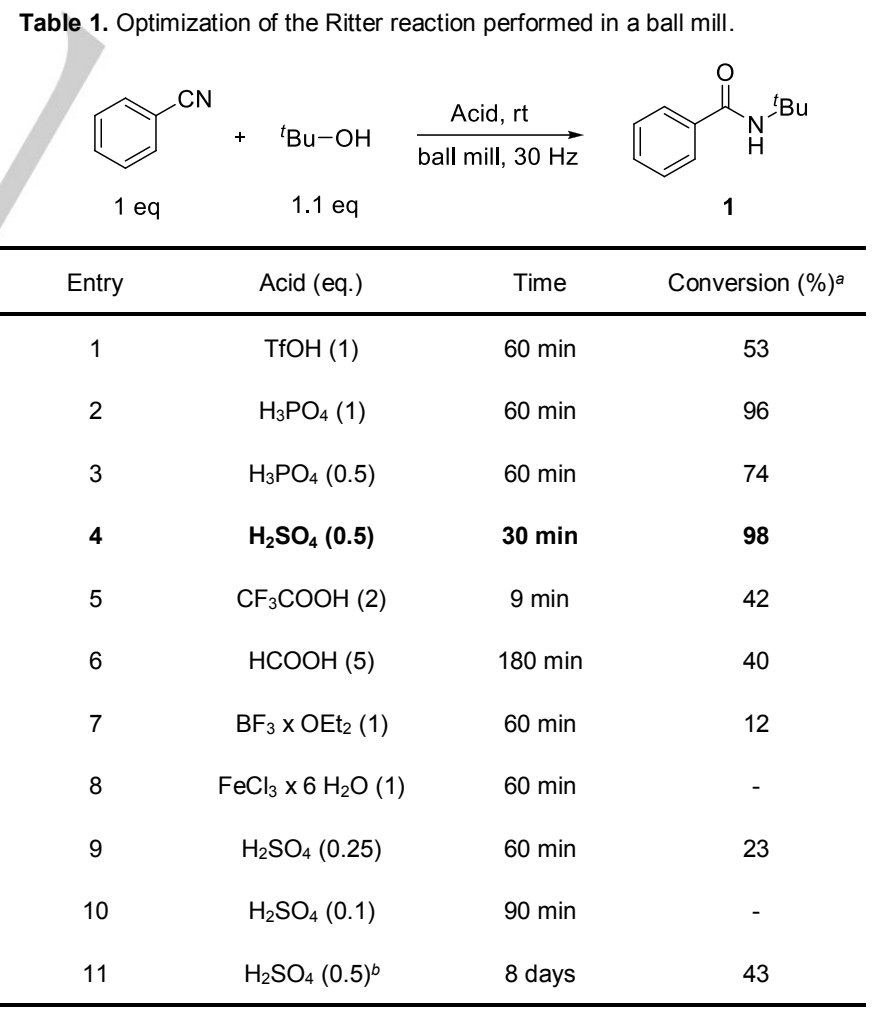

[a] Determined by ${ }^{1} \mathrm{H}$ NMR. [b] Reaction performed in a flask with stirring at room temperature. 
Due to the corrosive nature of strong acids, a Teflon ${ }^{\circledR}$ vial and a tungsten carbide ball (WC; $d=7 \mathrm{~mm}, \mathrm{~m}=4 \mathrm{~g}$ ) were used. In the first reaction, trifluoromethanesulfonic acid (1 eq.) was used. After 1 hour, the conversion to tert-butylbenzamide 1 was observed in $53 \%$ yield (Table 1, entry 1). Encouraged by this result, we approached catalyst screening and the study of reaction conditions. Employing phosphoric acid (1 eq.) as a catalyst resulted in $96 \%$ conversion after 60 min (Table 1, entry 2 ), while lowering its loading diminished conversion to $74 \%$ (Table 1, entry 3 ). On the other hand, $98 \%$ conversion was observed after 30 min of ball milling when 0.5 eq. of sulfuric acid was used as a catalyst (Table 1, entry 4).

Nucleophilic organic acids usually hinder the Ritter reaction, since they react with carbocations to yield esters. ${ }^{[19]}$ We decided here to test nucleophilic organic acids, and were pleased to see that reactions with trifluoroacetic and formic acid proceed without traces of ester (Table 1, entries 5 and 6). However, the transformation results in moderate conversions and requires longer reaction times with an excess of acid (2 eq. of formic acid and 5 eq. of trifluoroacetic acid, respectively). Lewis acids were also tested, but poor conversions were observed (Table 1, entries 7 and 8). Lowering the sulfuric acid loading increased reaction times and significantly decreased conversion (Table 1 , entries 9 and 10).

Hence, the optimized procedure employed solvent-free ball milling of a nitrile (1 eq.) and an alcohol (1.1 eq.) with sulfuric acid as a catalyst ( 0.5 eq.) at $30 \mathrm{~Hz}$ for $30 \mathrm{~min}$. In order to prove the efficiency of the mechanochemical Ritter reaction, optimized reaction conditions were used for a reaction in a flask with stirring at room temperature; after 8 days, the conversion was $43 \%$ (Table 1, entry 11).

Besides chemical parameters, technical and process parameters also require attention, such as the frequency of milling, size and number of balls, and the material from which balls are made of. ${ }^{[17 c]}$ Firstly, model reaction was performed at $25 \mathrm{~Hz}$ in order to test the influence of milling frequency. After 60 min, $86 \%$ conversion was observed (Table 2 , entry 2 ).

Table 2. Optimization of the ball mill conditions

\begin{tabular}{|c|c|c|c|c|c|}
\hline Entry & $\begin{array}{c}\text { Ball } \\
\text { material }\end{array}$ & $\begin{array}{l}\text { Number } \\
\text { of balls }\end{array}$ & Frequency & Time & $\begin{array}{c}\text { Conversion } \\
(\%)^{a}\end{array}$ \\
\hline 1 & wc & 1 & $30 \mathrm{~Hz}$ & $30 \mathrm{~min}$ & 98 \\
\hline 2 & WC & 1 & $25 \mathrm{~Hz}$ & $60 \mathrm{~min}$ & 86 \\
\hline 3 & Teflon $^{\circledR}$ & 1 & $30 \mathrm{~Hz}$ & $60 \mathrm{~min}$ & 73 \\
\hline 4 & corundum & 1 & $30 \mathrm{~Hz}$ & $60 \mathrm{~min}$ & 88 \\
\hline 5 & corundum & 2 & $30 \mathrm{~Hz}$ & $30 \mathrm{~min}$ & 94 \\
\hline
\end{tabular}

[a] Determined by ${ }^{1} \mathrm{H}$ NMR.
When larger, but lighter Teflon ${ }^{\circledR}$ ball $(\mathrm{d}=10 \mathrm{~mm}, \mathrm{~m}=1.76 \mathrm{~g})$ was used, the reaction yielded $73 \%$ conversion after $60 \mathrm{~min}$, while in the reaction with a corundum ball $(\mathrm{d}=6 \mathrm{~mm}, \mathrm{~m}=1 \mathrm{~g})$, $88 \%$ conversion was observed within the same period of time (Table 2, entries 3 and 4). Excellent conversion after 30 min was obtained with two corundum balls (Table 2 , entry 5 ), however, due to an excessive deterioration of the balls during milling, this protocol was not found suitable for the indicated reaction. Thus, best parameter combination for investigated process includes ball milling at $30 \mathrm{~Hz}$ with a single tungsten carbide ball. ${ }^{21]}$

With optimized reaction conditions at hand, we turned our attention to investigate substrate scope and reaction limitations. The first substrate scope comprised the Ritter reaction of various nitriles with tert-butanol as a model alcohol (Table 3).

Table 3. Substrate scope I: Nitriles. ${ }^{a}$

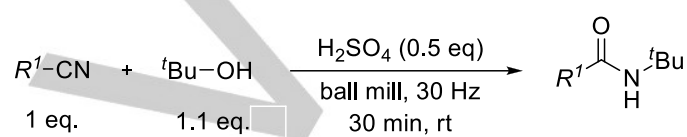

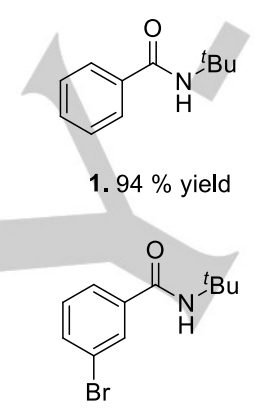

4. $76 \%$ yield $^{b}$<smiles>CCC(C)NC(=O)c1cc([N+](=O)[O-])ccc1Cl</smiles>

7. $82 \%$ yield $^{b}$<smiles>CCCCNC(=O)c1ccccc1C</smiles>

10. $44 \%$ yield $^{c}$

$$
{ }_{\mathrm{Me}}^{\mathrm{O}} \mathrm{N}^{t^{-B} \mathrm{Bu}}
$$

13. $93 \%$ yield

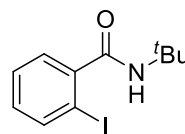

2. $79 \%$ yield $^{b}$<smiles>CCCCNC(=O)c1cccc(Cl)c1</smiles>

5. $86 \%$ yield $^{b}$<smiles>CCC(C)NC(=O)c1ccc(C(C)(C)C)cc1</smiles>

8. $61 \%$ yield $^{b, c}$<smiles>Cc1ccc(C(=O)NCC(C)(C)C)cc1</smiles>

11. $78 \%$ yield $^{b}$<smiles>CCCNC(=O)CCCl</smiles>

14. $91 \%$ yield<smiles>CC(C)CNC(=O)c1ccccc1F</smiles>

3. $88 \%$ yield
6. $73 \%$ yield $^{b}$<smiles>CC(C)CNC(=O)c1ccccc1C(F)(F)F</smiles>

9. $47 \%$ yield $^{b, c}$<smiles>COc1ccc(C(=O)NC(C)(C)C)cc1</smiles>

12. $63 \%$ yield $^{b, c}$<smiles>C=CC(=O)NC(C)(C)C</smiles>

15. $90 \%$ yield

[a] Yields are for isolated material. [b] LAG: $\mathrm{MeNO}_{2}$ (1 eq.). [c] $60 \mathrm{~min}$

When 2-iodobenzonitrile was used in the reaction, only traces of amide 2 were observed. Since the reaction mixture was a solid, we presumed that the carbocation could not be stabilized long enough for the reaction to occur. Therefore, liquid-assisted grinding $(\mathrm{LAG})^{[22]}$ was performed with a polar, non-nucleophilic 
additive possessing the ability to stabilize the carbocation. Indeed, upon the addition of nitromethane (1 eq., $\eta=0.26 \mu \mathrm{L} /$ $\mathrm{mg}),{ }^{[23]}$ the reaction rapidly improved to $79 \%$ isolated yield (Table 3, 2). The transformation maintained its effectiveness upon introducing various halogen atoms on different positions throughout the aromatic ring (Table 3, 3-6). The reaction was tolerant of other aromatic ring deactivating groups (nitro group 7 , ester group 8, $\mathrm{CF}_{3}$ group 9), as well as of aromatic ring activating groups (methyl group in ortho 10 and para 11 positions, methoxy group 12). Moderate isolated yields were observed with methyl and trifluoromethyl ortho substituted benzonitriles 9 and 10, presumably due to steric hindrances. Changing aromatic nitriles with alkyl and allyl nitriles was also successful, generating respective amides 13-15 in excellent yields.

With a survey of reactivity of various nitriles in the mechanochemical Ritter reaction conducted, the scope of secondary and tertiary alcohols was explored using acetonitrile and benzonitrile as nucleophiles (Table 4).

Table 4. Substrate scope II: Alcohols. ${ }^{a}$

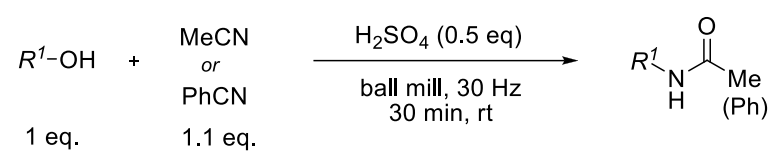

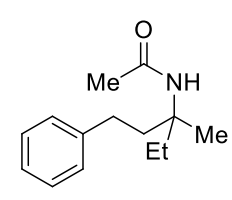

16. $77 \%$ yield<smiles>CC(=O)NC(C)(C)Cc1ccccc1</smiles>

19. $74 \%$ yield $^{b}$

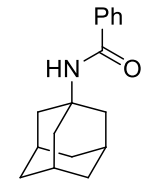

17. $85 \%$ yield<smiles>CC(=O)NC(C)c1ccccc1</smiles>

20. $84 \%$ yield $^{c}$

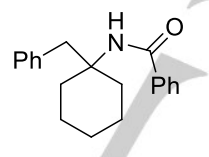

18. $81 \%$ yield

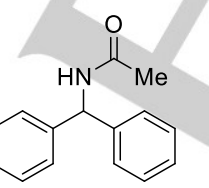

21. $74 \%$ yield $^{d}$

[a] Yields are for isolated material. [b] $\mathrm{MeCN}$ (5 eq.). [c] $\mathrm{MeCN}$ (5 eq.) $\mathrm{H}_{2} \mathrm{SO}_{4}$ (2 eq.), 60 min. [d] LAG: $\mathrm{MeNO}_{2}$ (1 eq.), 60 min.

The reaction proved to be effective, furnishing amides 16-18 from tertiary alcohols in high yields. However, under optimized reaction conditions, amide 19 was obtained only in traces; the major product was an olefin formed by intramolecular trapping of the carbocation. ${ }^{[24]}$ Since the intramolecular trapping of the carbocation is in competition with its trapping by a nitrile group, we reasoned that using an excess of a nitrile would govern the reaction towards the amide. After systematic increase of the nitrile component, the amide 19 was afforded as a sole product with 5 eq. of acetonitrile in $74 \%$ yield.

The effectiveness of the reaction was also tested on secondary alcohols. As with the previous example, under optimized reaction conditions 1-phenyletanol afforded elimination product styrene, rather than amide 20. The increase of the nitrile amount did not change the reaction outcome. In addition to 5 eq. of acetonitrile, 2 eq. of sulfuric acid and longer reaction time were required to afford desired amide in $84 \%$ isolated yield. The Ritter reaction between diphenylmethanol and acetonitrile to afford amide $\mathbf{2 1}$ required longer reaction time, most likely due to sterical hindrances of two phenyl groups.

It is worth noting that many of prepared amides have been obtained for the first time, while a number of others by employing Ritter reaction, rather than more expensive methods mentioned earlier.

The scale-up of the developed protocol was also explored. A gram-scale reaction between benzonitrile and tert-butanol afforded tert-butylbenzamide 1 in $84 \%$ isolated yield (Scheme 1).

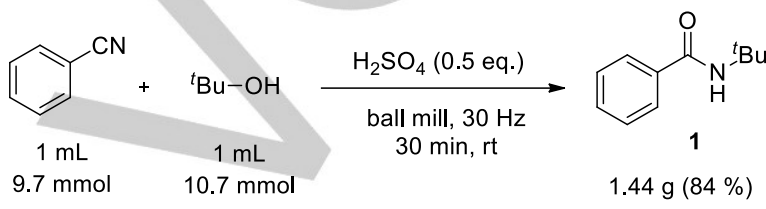

Scheme 1. Scale-up reaction.

\section{Conclusion}

In conclusion, we have developed an efficient mechanochemical Ritter reaction between nitriles and alcohols under mild conditions utilizing a Brønsted acid catalyst. The transformation is fast, proceeds at room temperature, and is tolerant of various functionalized nitriles, as well as secondary and tertiary alcohols. This process offers a rapid approach to functionalized amides, and may find application in the synthesis of complex frameworks and natural product analogues comprising sensitive functional groups.

\section{Experimental Section}

General procedure for the synthesis of amides $1-15$. Teflon ${ }^{\circledR}$ grinding vial $(10 \mathrm{~mL})$ with a single tungsten carbide ball $(\mathrm{d}=7 \mathrm{~mm}, \mathrm{~m}=4 \mathrm{~g})$ was charged with a nitrile (1 eq.), tert-butanol (1.1 eq.) and sulfuric acid (0.5 eq.), with or without nitromethane (1 eq.), and mixed in a ball mill for 30 $\min$ at $30 \mathrm{~Hz}$. The reaction mixture was dissolved in ethyl acetate, and washed with sat. $\mathrm{NaHCO}_{3}$, water and brine. Amide was recrystallized from ethyl acetate/hexane or purified by flash column chromatography.

Procedure for the scale-up synthesis of 1 . Teflon ${ }^{\circledR}$ grinding vial (10 $\mathrm{mL})$ with a single tungsten carbide ball $(\mathrm{d}=7 \mathrm{~mm}, \mathrm{~m}=4 \mathrm{~g})$ was charged with benzonitrile $(1 \mathrm{ml}, 9.71 \mathrm{mmol})$, tert-butanol $(1 \mathrm{~mL}, 10.68 \mathrm{mmol})$ and sulfuric acid $(249 \mu \mathrm{L}, 4.68 \mathrm{mmol})$, and mixed in a ball mill for $30 \mathrm{~min}$ at 30 $\mathrm{Hz}$. The reaction mixture was dissolved in ethyl acetate, and washed with sat. $\mathrm{NaHCO}_{3}$, water and brine. Amide 1 was recrystallized from ethyl acetate/hexane and obtained as a white solid (1.44 g, $84 \%$ ).

$N$-(tert-butyl)benzamide (1). ${ }^{[25]}$ Benzonitrile $(64 \mu \mathrm{L}, 0.62 \mathrm{mmol})$ afforded amide 1 as a white solid. Purified by recrystallization (ethyl acetate/hexane). Yield: $103 \mathrm{mg}, 94 \% .{ }^{1} \mathrm{H}$ NMR $\left(300 \mathrm{MHz}, \mathrm{CDCl}_{3}\right) \delta 7.80$ - $7.67(\mathrm{~m}, 2 \mathrm{H}), 7.53-7.34(\mathrm{~m}, 3 \mathrm{H}), 6.01(\mathrm{br} \mathrm{s}, 1 \mathrm{H}), 1.49(\mathrm{~s}, 9 \mathrm{H}) .{ }^{13} \mathrm{C}$ 
NMR $\left(75 \mathrm{MHz}, \mathrm{CDCl}_{3}\right) \delta 166.9,135.9,131.1,128.5,126.7,51.6,28.9$. ESI-MS: $m / z 178[M+H]^{+}$.

$\mathbf{N}$-(tert-butyl)-2-iodobenzamide (2). ${ }^{[26]}$ 2-iodobenzonitrile $(70 \mathrm{mg}, 0.31$ $\mathrm{mmol}$ ) afforded amide 2 as a white solid. Purified by flash column chromatography (petrol-ethyl acetate 5:1). Yield: $74 \mathrm{mg}, 79 \% . \eta=0.26$ $\mu \mathrm{L} / \mathrm{mg} .{ }^{1} \mathrm{H}$ NMR $\left(600 \mathrm{MHz}, \mathrm{CDCl}_{3}\right) \delta 7.84-7.80(\mathrm{~m}, 1 \mathrm{H}), 7.40-7.31(\mathrm{~m}$, $2 \mathrm{H}), 7.06$ (td, $J=7.9,2.0 \mathrm{~Hz}, 1 \mathrm{H}), 5.55(\mathrm{br} \mathrm{s}, 1 \mathrm{H}), 1.48(\mathrm{~s}, 9 \mathrm{H}) .{ }^{13} \mathrm{C}$ NMR $\left(151 \mathrm{MHz}, \mathrm{CDCl}_{3}\right) \delta 168.7,143.3,139.7,130.7,128.1,92.4,52.2,28.7$. ESI-MS: $m / z 304[\mathrm{M}+\mathrm{H}]^{+}$

$\mathbf{N}$-(tert-butyl)-2-fluorobenzamide (3). ${ }^{[26]}$ 2-fluorobenzonitrile $(68 \mu \mathrm{L}$, $0.62 \mathrm{mmol}$ ) afforded amide 3 as a yellow oil. Purified by flash column chromatography (petrol-ethyl acetate 5:1). Yield: $106 \mathrm{mg}, 88 \%$. ${ }^{1} \mathrm{H}$ NMR $\left(600 \mathrm{MHz}, \mathrm{CDCl}_{3}\right) \delta 8.05(\mathrm{td}, J=8.0,1.8 \mathrm{~Hz}, 1 \mathrm{H}), 7.47-7.39(\mathrm{~m}, 1 \mathrm{H})$ $7.29-7.20(\mathrm{~m}, 1 \mathrm{H}), 7.12-7.05(\mathrm{~m}, 1 \mathrm{H}), 6.58(\mathrm{br} \mathrm{s}, 1 \mathrm{H}), 1.47(\mathrm{~s}, 9 \mathrm{H})$. $\left.{ }^{13} \mathrm{C} \mathrm{NMR} \mathrm{(151} \mathrm{MHz,} \mathrm{CDCl}_{3}\right) \delta 162.3,160.4(\mathrm{~d}, J=245.9 \mathrm{~Hz}), 132.8$ (d, $J$ $=9.2 \mathrm{~Hz}), 131.8(\mathrm{~d}, J=2.1 \mathrm{~Hz}), 124.7(\mathrm{~d}, J=3.3 \mathrm{~Hz}), 122.4(\mathrm{~d}, J=11.8$ $\mathrm{Hz}), 115.9(\mathrm{~d}, J=25.3 \mathrm{~Hz}), 51.8,28.9$. ESI-MS: $\mathrm{m} / \mathrm{z} 196[\mathrm{M}+\mathrm{H}]^{+}$.

3-bromo-N-(tert-butyl)benzamide (4). 3-bromobenzonitrile (90 mg, 0.49 $\mathrm{mmol}$ ) afforded amide 4 as a white solid. Purified by recrystallization (ethyl acetate/hexane). Yield: $95 \mathrm{mg}, 76 \% . \eta=0.30 \mu \mathrm{L} / \mathrm{mg} .{ }^{1} \mathrm{H}$ NMR $\left(600 \mathrm{MHz}, \mathrm{CDCl}_{3}\right) \delta 7.85(\mathrm{t}, J=1.7 \mathrm{~Hz}, 1 \mathrm{H}), 7.66-7.61(\mathrm{~m}, 1 \mathrm{H}), 7.61-$ $7.57(\mathrm{~m}, 1 \mathrm{H}), 7.31-7.25(\mathrm{~m}, 1 \mathrm{H}), 5.91$ (br s, 1H), $1.47(\mathrm{~s}, 9 \mathrm{H}) .{ }^{13} \mathrm{C}$ NMR $\left(151 \mathrm{MHz}, \mathrm{CDCl}_{3}\right) \delta 165.4,138.0,134.0,130.0,129.9,125.4,122.6,51.9$ 28.8. $\mathrm{mp} 106.3$ - $107.1{ }^{\circ} \mathrm{C}$. IR (KBr): $3273,3069,2984,1637,1542$, 1317, $1069 \mathrm{~cm}^{-1}$. HRMS (ESI): found 256.0337; $\mathrm{C}_{11} \mathrm{H}_{14} \mathrm{BrNO}[\mathrm{M}+\mathrm{H}]^{+}$ requires 256.0331 .

$\mathbf{N}$-(tert-butyl)-3-chlorobenzamide (5). ${ }^{[15]} 3$-chlorobenzonitrile $(85 \mathrm{mg}$, $0.62 \mathrm{mmol}$ ) afforded amide 5 as a white solid. Purified by recrystallization (ethyl acetate/hexane). Yield: $111 \mathrm{mg}, 86 \% . \eta=0.36 \mu \mathrm{L} / \mathrm{mg} .{ }^{1} \mathrm{H}$ NMR $\left(600 \mathrm{MHz}, \mathrm{CDCl}_{3}\right) \delta 7.69$ (t, $\left.J=1.6 \mathrm{~Hz}, 1 \mathrm{H}\right), 7.56(\mathrm{t}, J=8.7 \mathrm{~Hz}, 1 \mathrm{H}), 7.44$ $-7.39(\mathrm{~m}, 1 \mathrm{H}), 7.32(\mathrm{t}, J=7.8 \mathrm{~Hz}, 1 \mathrm{H}), 5.99(\mathrm{br} \mathrm{s}, 1 \mathrm{H}), 1.46(\mathrm{~s}, 9 \mathrm{H}) \cdot{ }^{13} \mathrm{C}$ NMR $\left(151 \mathrm{MHz}, \mathrm{CDCl}_{3}\right) \delta 165.5,137.8,134.6,131.0,129.7,127.1$, 124.9, 51.9, 28.8. ESI-MS: $m / z 212[\mathrm{M}+\mathrm{H}]^{+}$.

\section{3-bromo- $\mathrm{N}$-(tert-butyl)-5-fluorobenzamide (6).}

3-bromo-5-fluorobenzonitrile $(100 \mathrm{mg}, 0.50 \mathrm{mmol}$ ) afforded amide 6 as a white solid. Purified by flash column chromatography (petrol-ethyl acetate 5:1). Yield: $100 \mathrm{mg}, 73 \% . \eta=0.28 \mu \mathrm{L} / \mathrm{mg} .{ }^{1} \mathrm{H}$ NMR $\left(300 \mathrm{MHz}, \mathrm{CDCl}_{3}\right) \delta$ $7.59(\mathrm{~s}, 1 \mathrm{H}), 7.40-7.29(\mathrm{~m}, 2 \mathrm{H}), 5.99(\mathrm{br} \mathrm{s}, 1 \mathrm{H}), 1.46(\mathrm{~s}, 9 \mathrm{H}) .{ }^{13} \mathrm{C}$ NMR $\left(75 \mathrm{MHz}, \mathrm{CDCl}_{3}\right) \delta 164.2$ (d, $\left.J=2.4 \mathrm{~Hz}\right), 162.5(\mathrm{~d}, J=252.5 \mathrm{~Hz}), 139.5$ $(\mathrm{d}, J=7.0 \mathrm{~Hz}), 125.7(\mathrm{~d}, J=3.2 \mathrm{~Hz}), 122.7(\mathrm{~d}, J=9.2 \mathrm{~Hz}), 121.5(\mathrm{~d}, J=$ $24.5 \mathrm{~Hz}), 113.3(\mathrm{~d}, J=22.7 \mathrm{~Hz}), 52.1$, 28.7. $\mathrm{mp} 102.0-102.5^{\circ} \mathrm{C}$. IR $(\mathrm{KBr}): 3348,3085,2970,1645,1579,1537,1313,1220,1090 \mathrm{~cm}^{-1}$. HRMS (ESI): found 274.0247; $\mathrm{C}_{11} \mathrm{H}_{13} \mathrm{BrFNO}[\mathrm{M}+\mathrm{H}]^{+}$requires 274.0237 .

\section{$\mathrm{N}$-(tert-butyl)-2-chloro-5-nitrobenzamide (7).}

2-chloro-5-nitrobenzonitrile $(57 \mathrm{mg}, 0.31 \mathrm{mmol}$ ) afforded amide 7 as a white solid. Purified by recrystallization (ethyl acetate/hexane). Yield: 67 $\mathrm{mg}, 82 \% . \eta=0.30 \mu \mathrm{L} / \mathrm{mg} .{ }^{1} \mathrm{H}$ NMR $\left(600 \mathrm{MHz}, \mathrm{CDCl}_{3}\right) \delta 8.37(\mathrm{~d}, J=2.7$ $\mathrm{Hz}, 1 \mathrm{H}), 8.20-8.11(\mathrm{~m}, 1 \mathrm{H}), 7.55(\mathrm{~d}, J=8.8 \mathrm{~Hz}, 1 \mathrm{H}), 5.98(\mathrm{br} \mathrm{s}, 1 \mathrm{H})$ $1.48(\mathrm{~s}, 9 \mathrm{H}) .{ }^{13} \mathrm{C}$ NMR $\left(151 \mathrm{MHz}, \mathrm{CDCl}_{3}\right) \delta 163.6,146.5,137.8,137.4$ 131.2, 125.1, 124.6, 52.8, 28.7. mp $113.0-113.9^{\circ} \mathrm{C}$. IR (KBr): 3308 3109, 2964, 1672, 1648, 1527, 1347, 1307, $1050 \mathrm{~cm}^{-1}$. HRMS (ESI): found 257.0684; $\mathrm{C}_{11} \mathrm{H}_{13} \mathrm{ClN}_{2} \mathrm{O}_{3}[\mathrm{M}+\mathrm{H}]^{+}$requires 257.0688 .

Methyl 4-(tert-butylcarbamoyl)benzoate (8). ${ }^{[8]}$ Methyl 4-cyanobenzoate ( $81 \mathrm{mg}, 0.50 \mathrm{mmol}$ ) afforded amide 8 as a white solid. Purified by flash column chromatography (petrol-ethyl acetate 5:1). Yield: $72 \mathrm{mg}, 61 \%$. $\eta$ $=0.33 \mu \mathrm{L} / \mathrm{mg} .{ }^{1} \mathrm{H}$ NMR $\left(300 \mathrm{MHz}, \mathrm{CDCl}_{3}\right) \delta 8.08-7.99(\mathrm{~m}, 2 \mathrm{H}), 7.81-$ $7.71(\mathrm{~m}, 2 \mathrm{H}), 6.12(\mathrm{br} \mathrm{s}, 1 \mathrm{H}), 3.92(\mathrm{~s}, 3 \mathrm{H}), 1.48(\mathrm{~s}, 9 \mathrm{H}) .{ }^{13} \mathrm{C}$ NMR $(75$ $\left.\mathrm{MHz}, \mathrm{CDCl}_{3}\right) \delta 166.3,166.1,139.9,132.2,129.7,126.8,52.3,51.9,28.8$. ESI-MS: $m / z 236[\mathrm{M}+\mathrm{H}]^{+}, 258[\mathrm{M}+\mathrm{Na}]^{+}$
$\mathbf{N}$-(tert-butyl)-2-(trifluoromethyl)benzamide (9).

2-(trifluoromethyl)benzonitrile ( $66 \mu \mathrm{L}, 0.50 \mathrm{mmol}$ ) afforded amide 9 as a white solid. Purified by flash column chromatography (petrol-ethyl acetate 5:1). Yield: $57 \mathrm{mg}, 47 \%$. ${ }^{1} \mathrm{H} \mathrm{NMR}\left(300 \mathrm{MHz}, \mathrm{CDCl}_{3}\right) \delta 7.66(\mathrm{~d}, J=7.7 \mathrm{~Hz}$, $2 \mathrm{H}), 7.61-7.44(\mathrm{~m}, 3 \mathrm{H}), 5.55(\mathrm{br} \mathrm{s}, 1 \mathrm{H}), 1.45(\mathrm{~s}, 9 \mathrm{H}) .{ }^{13} \mathrm{C} \mathrm{NMR}(75 \mathrm{MHz}$ $\left.\mathrm{CDCl}_{3}\right) \delta 167.0,137.2,132.0,129.4,128.6,126.9(\mathrm{~d}, J=31.7 \mathrm{~Hz}), 126.2$ (q, $J=5.1 \mathrm{~Hz}), 123.8(\mathrm{~d}, J=273.8 \mathrm{~Hz}), 52.2,28.5 \mathrm{mp} 107.9-108.4^{\circ} \mathrm{C}$ IR (KBr): $3300,2973,1646,1543,1316,1125 \mathrm{~cm}^{-1}$. HRMS (ESI): found 246.1110; $\mathrm{C}_{12} \mathrm{H}_{14} \mathrm{~F}_{3} \mathrm{NO}[\mathrm{M}+\mathrm{H}]^{+}$requires 246.1100 .

$\mathbf{N}$-(tert-butyl)-2-methylbenzamide (10). o-toluonitrile (73 $\mu \mathrm{L}, 0.62 \mathrm{mmol})$ afforded amide 10 as a colourless oil. Purified by flash column chromatography (petrol-ethyl acetate 5:1). Yield: $52 \mathrm{mg}, 44 \% .{ }^{1} \mathrm{H}$ NMR $\left(300 \mathrm{MHz}, \mathrm{CDCl}_{3}\right) \delta 7.35-7.23(\mathrm{~m}, 2 \mathrm{H}), 7.22-7.11(\mathrm{~m}, 2 \mathrm{H}), 5.59(\mathrm{br} \mathrm{s}$, $1 \mathrm{H}), 2.42(\mathrm{~s}, 3 \mathrm{H}), 1.46(\mathrm{~s}, 9 \mathrm{H}) .{ }^{13} \mathrm{C} \mathrm{NMR}\left(75 \mathrm{MHz}, \mathrm{CDCl}_{3}\right) \delta 169.7,137.9$ 135.4, 130.8, 129.4, 126.4, 125.6, 51.7, 28.9, 19.5. IR (KBr): 3307, 2923, $1647,1541,1316 \mathrm{~cm}^{-1}$. HRMS (ESI): found 192.1390; $\mathrm{C}_{12} \mathrm{H}_{17} \mathrm{NO}[\mathrm{M}+\mathrm{H}]^{+}$ requires 192.1383 .

$N$-(tert-butyl)-4-methylbenzamide (11). ${ }^{[8]} p$-toluonitrile $(58 \mathrm{mg}, 0.50$ $\mathrm{mmol}$ ) afforded amide 11 as a white solid. Purified by flash column chromatography (petrol-ethyl acetate $5: 1$ ). Yield: $74 \mathrm{mg}, 78 \% . \eta=0.40$ $\mu \mathrm{L} / \mathrm{mg} .{ }^{1} \mathrm{H}$ NMR $\left(300 \mathrm{MHz}, \mathrm{CDCl}_{3}\right) \delta 7.61(\mathrm{~d}, J=8.1 \mathrm{~Hz}, 2 \mathrm{H}), 7.20$ (d, $J$ $=7.9 \mathrm{~Hz}, 2 \mathrm{H}), 5.92(\mathrm{br} \mathrm{s}, 1 \mathrm{H}), 2.38(\mathrm{~s}, 3 \mathrm{H}), 1.46(\mathrm{~s}, 9 \mathrm{H}) .{ }^{13} \mathrm{C}$ NMR $(75$ $\left.\mathrm{MHz}, \mathrm{CDCl}_{3}\right) \delta 166.8,141.4,133.1,129.1,126.7,51.5,28.9,21.4$. ESIMS: $m / z 192[\mathrm{M}+\mathrm{H}]^{+}$.

$\mathbf{N}$-(tert-butyl)-4-methoxybenzamide (12). ${ }^{[8]}$ 4-methoxybenzonitrile $(67$ $\mathrm{mg}, 0.50 \mathrm{mmol}$ ) afforded amide 12 as a white solid. Purified by flash column chromatography (petrol-ethyl acetate $5: 1$ ). Yield: $65 \mathrm{mg}, 63 \%$. $\eta$ $=0.37 \mu \mathrm{L} / \mathrm{mg}$. ${ }^{1} \mathrm{H}$ NMR $\left(300 \mathrm{MHz}, \mathrm{CDCl}_{3}\right) \delta 7.74-7.66(\mathrm{~m}, 2 \mathrm{H}), 6.96-$ $6.86(\mathrm{~m}, 2 \mathrm{H}), 5.88(\mathrm{br} \mathrm{s}, 1 \mathrm{H}), 3.85(\mathrm{~s}, 3 \mathrm{H}), 1.48(\mathrm{~s}, 9 \mathrm{H}) .{ }^{13} \mathrm{C}$ NMR $(75$ $\left.\mathrm{MHz}, \mathrm{CDCl}_{3}\right) \delta 166.4,161.9,128.4,128.2,113.6,55.4,51.5,29.0 . \mathrm{ESI}-$ MS: $m / z 208[\mathrm{M}+\mathrm{H}]^{+}$

$N$-(tert-butyl)acetamide (13). ${ }^{[6 b]}$ Acetonitrile $(32 \mu \mathrm{L}, 0.62 \mathrm{mmol})$ afforded amide 13 as a white solid. Purified by recrystallization (ethyl acetate/hexane). Yield: $67 \mathrm{mg}, 93 \%$. ${ }^{1} \mathrm{H}$ NMR $\left(600 \mathrm{MHz}, \mathrm{CDCl}_{3}\right) \delta 5.22$ (br s, 1H), 1.91 (s, 3H), $1.36(\mathrm{~s}, 9 \mathrm{H}) \cdot{ }^{13} \mathrm{C} \mathrm{NMR}\left(151 \mathrm{MHz}, \mathrm{CDCl}_{3}\right) \delta 169.4$, 51.2, 28.8, 24.5. ESI-MS: $\mathrm{m} / \mathrm{z} 116[\mathrm{M}+\mathrm{H}]^{+}$.

$\mathbf{N}$-(tert-butyl)-3-chloropropanamide (14). 2-chloropropionitrile (48 $\mu \mathrm{L}$, $0.62 \mathrm{mmol}$ ) afforded amide 14 as a white solid. Purified by recrystallization (ethyl acetate/hexane). Yield: $92 \mathrm{mg}, 91 \%$. ${ }^{1} \mathrm{H}$ NMR $(600$ $\mathrm{MHz}, \mathrm{CDCl}_{3}$ ) $\delta 5.67$ (br s, $1 \mathrm{H}$ ), $3.78(\mathrm{t}, J=6.5 \mathrm{~Hz}, 2 \mathrm{H}), 2.55$ (t, $J=6.5 \mathrm{~Hz}$, $2 \mathrm{H}), 1.36(\mathrm{~s}, 9 \mathrm{H}) .{ }^{13} \mathrm{C} \mathrm{NMR}\left(151 \mathrm{MHz}, \mathrm{CDCl}_{3}\right) \delta 168.8,51.6,40.4,40.3$, 28.7. $\mathrm{mp} 90.6-91.4{ }^{\circ} \mathrm{C}$. IR (KBr): 3284, 3091, 2970, 1647, 1566, 1362 $\mathrm{cm}^{-1}$. HRMS (ESI): found 164.0829; $\mathrm{C}_{7} \mathrm{H}_{14} \mathrm{CINO}[\mathrm{M}+\mathrm{H}]^{+}$requires 164.0837

$\mathrm{N}$-(tert-butyl)acrylamide (15).[6b] Acrylonitrile (41 $\mu \mathrm{L}, 0.62 \mathrm{mmol})$ afforded amide 15 as a white solid. Purified by recrystallization (ethyl acetate/hexane). Yield: $70 \mathrm{mg}, 90 \%$. ${ }^{1} \mathrm{H}$ NMR $\left(600 \mathrm{MHz}, \mathrm{CDCl}_{3}\right) \delta 6.22$ (dd, $J=16.9,1.4 \mathrm{~Hz}, 1 \mathrm{H}), 6.04(\mathrm{dd}, J=16.9,10.2 \mathrm{~Hz}, 1 \mathrm{H}), 5.56(\mathrm{dt}, J=$ $8.8,4.4 \mathrm{~Hz}, 1 \mathrm{H}), 5.52(\mathrm{~s}, 1 \mathrm{H}), 1.40(\mathrm{~s}, 9 \mathrm{H}) .{ }^{13} \mathrm{C} \mathrm{NMR}\left(151 \mathrm{MHz}, \mathrm{CDCl}_{3}\right) \delta$ 164.8, 132.1, 125.5, 51.3, 28.8. ESI-MS: $m / z 128[\mathrm{M}+\mathrm{H}]^{+}$

General procedure for the synthesis of amides 16-21. Teflon ${ }^{\circledR}$ grinding vial $(10 \mathrm{~mL})$ with a single tungsten carbide ball $(\mathrm{d}=7 \mathrm{~mm}, \mathrm{~m}=4$ g) was charged with an alcohol (1 eq.), acetonitrile or benzonitrile (1.1 eq.) and sulfuric acid ( 0.5 eq.), with or without nitromethane $(1 \mathrm{eq})$, and mixed in a ball mill for $30 \mathrm{~min}$ at $30 \mathrm{~Hz}$. The reaction mixture was dissolved in ethyl acetate, and washed with sat. $\mathrm{NaHCO}_{3}$, water and brine. Amides was recrystallized from ethyl acetate/hexane or purified by flash column chromatography 
$N$-(3-methyl-1-phenylpentan-3-yl)acetamide (16). 3-methyl-1-phenylpentan-3-ol (colorless oil; $80 \mathrm{mg}, 0.45 \mathrm{mmol}$ ) afforded amide 16 as a colourless oil. Purified by flash column chromatography (petrol-ethyl acetate 3:1). Yield: $75 \mathrm{mg}, 77 \% .{ }^{1} \mathrm{H}$ NMR $\left(600 \mathrm{MHz}, \mathrm{CDCl}_{3}\right) \delta 7.27-$ $7.22(\mathrm{~m}, 2 \mathrm{H}), 7.19-7.12(\mathrm{~m}, 3 \mathrm{H}), 5.54(\mathrm{br} \mathrm{s}, 1 \mathrm{H}), 2.55(\mathrm{t}, J=8.5 \mathrm{~Hz}, 2 \mathrm{H})$ $2.16-2.07(\mathrm{~m}, 1 \mathrm{H}), 1.90(\mathrm{~s}, 3 \mathrm{H}), 1.95-1.82(\mathrm{~m}, 2 \mathrm{H}), 1.69-1.60(\mathrm{~m}$, $1 \mathrm{H}), 1.28(\mathrm{~s}, 3 \mathrm{H}), 0.85(\mathrm{t}, J=7.5 \mathrm{~Hz}, 3 \mathrm{H}) .{ }^{13} \mathrm{C}$ NMR $\left(151 \mathrm{MHz}, \mathrm{CDCl}_{3}\right) \delta$ $169.8,142.4,128.4,128.4,125.7,56.7,39.7,31.0,30.4,24.2,23.8,8.0$. IR (KBr): 3314, 3061, 2970, 1657, 1554, 1452, 1372, 740, $696 \mathrm{~cm}^{-1}$. HRMS (ESI): found 220.1707; $\mathrm{C}_{14} \mathrm{H}_{21} \mathrm{NO}[\mathrm{M}+\mathrm{H}]^{+}$requires 220.1696

$N$-((3s,5s,7s)-adamantan-1-yl)benzamide (17). ${ }^{\text {7a] }}$ Adamantane-1-ol (35 $\mathrm{mg}, 0.23 \mathrm{mmol}$ ) afforded amide 17 as a white solid. Purified by recrystallization (ethyl acetate/hexane). Yield: $45 \mathrm{mg}, 85 \%$. ${ }^{1} \mathrm{H}$ NMR (300 $\left.\mathrm{MHz}, \mathrm{CDCl}_{3}\right) \delta 7.76-7.65(\mathrm{~m}, 2 \mathrm{H}), 7.50-7.34(\mathrm{~m}, 3 \mathrm{H}), 5.83(\mathrm{br} \mathrm{s}, 1 \mathrm{H})$, 2.13 (br s, 9H), 1.72 (br s, 6H). ${ }^{13} \mathrm{C} \mathrm{NMR}\left(75 \mathrm{MHz}, \mathrm{CDCl}_{3}\right) \delta 166.6,136.1$ 131.0, 128.4, 126.7, 52.3, 41.7, 36.4, 29.5. ESI-MS: $\mathrm{m} / \mathrm{z} 256[\mathrm{M}+\mathrm{H}]^{+}$.

$\mathbf{N}$-(1-benzylcyclohexyl)benzamide (18). 1-benzyl-cyclohexanol (104 mg $0.50 \mathrm{mmol}$ ) afforded amide 18 as a white solid. Purified by flash column chromatography (petrol-ethyl acetate 5:1). Yield: $114 \mathrm{mg}, 81 \%$. ${ }^{1} \mathrm{H}$ NMR $\left(600 \mathrm{MHz}, \mathrm{CDCl}_{3}\right) \delta 7.65(\mathrm{~d}, J=7.4 \mathrm{~Hz}, 2 \mathrm{H}), 7.45(\mathrm{t}, J=7.4 \mathrm{~Hz}, 1 \mathrm{H})$, $7.38(\mathrm{t}, J=7.6 \mathrm{~Hz}, 2 \mathrm{H})(\mathrm{H} 9, \mathrm{H} 10), 7.22-7.11(\mathrm{~m}, 5 \mathrm{H}), 5.44(\mathrm{br} \mathrm{s}, 1 \mathrm{H})$, $3.19(\mathrm{~s}, 2 \mathrm{H}), 2.22(\mathrm{~d}, J=12.3 \mathrm{~Hz}, 2 \mathrm{H}), 1.63(\mathrm{~d}, J=10.2 \mathrm{~Hz}, 3 \mathrm{H}), 1.52-$ $1.39(\mathrm{~m}, 4 \mathrm{H}), 1.34-1.25(\mathrm{~m}, 1 \mathrm{H}) .{ }^{13} \mathrm{C}$ NMR $\left(151 \mathrm{MHz}, \mathrm{CDCl}_{3}\right) \delta 167.5$, 137.6, 136.4, 131.1, 130.7, 128.6, 127.8, 126.7, 126.2, 56.7, 43.7, 35.0, 25.7, 21.9. mp $99.4-100.3^{\circ} \mathrm{C}$. IR (KBr): $3371,3055,2923,1635,1533$ $1449 \mathrm{~cm}^{-1}$. HRMS (ESI): found 294.1866; $\mathrm{C}_{20} \mathrm{H}_{23} \mathrm{NO}[\mathrm{M}+\mathrm{H}]^{+}$requires 294.1852.

\section{$N$-(2-methyl-1-phenylpropan-2-yl)acetamide (19)}

2-methyl-1-phenylpropan-2-ol (colorless oil; $75 \mathrm{mg}, 0.50 \mathrm{mmol}$ ) afforded amide 19 as a white solid. Purified by flash column chromatography (petrol-ethyl acetate 3:1). Yield: $70 \mathrm{mg}, 74 \% .{ }^{1} \mathrm{H}$ NMR $(300 \mathrm{MHz}$, $\left.\mathrm{CDCl}_{3}\right) \delta 7.32-7.17(\mathrm{~m}, 3 \mathrm{H}), 7.16-7.09(\mathrm{~m}, 2 \mathrm{H}), 5.18$ (br s, 1H), 3.04 (s, 2H), $1.88(\mathrm{~s}, 3 \mathrm{H}), 1.31$ (s, 6H). ${ }^{13} \mathrm{C}$ NMR $\left(75 \mathrm{MHz}, \mathrm{CDCl}_{3}\right) \delta 169.8$, 138.1, 130.5, 127.9, 126.3, 54.0, 44.6, 27.4, 24.5. mp 92.9-93.7 ${ }^{\circ} \mathrm{C}$. IR (KBr): 3283, 3088, 2958, 1644, 1564, $1362 \mathrm{~cm}^{-1}$. HRMS (ESI): found 192.1384; $\mathrm{C}_{12} \mathrm{H}_{17} \mathrm{NO}[\mathrm{M}+\mathrm{H}]^{+}$requires 192.1383

$\mathbf{N}$-(1-phenylethyl)acetamide (20). ${ }^{[6 c]}$ 1-phenylethanol (colorless oil; 61 $\mathrm{mg}, 0.50 \mathrm{mmol}$ ) afforded amide 20 as a white solid. Purified by flash column chromatography (petrol-ethyl acetate 3:1). Yield: $68 \mathrm{mg}, 84 \%$. ${ }^{1} \mathrm{H}$ NMR $\left(300 \mathrm{MHz}, \mathrm{CDCl}_{3}\right) \delta 7.43-7.20(\mathrm{~m}, 5 \mathrm{H}), 5.81$ (br s, 1H), $5.13(\mathrm{p}$ $J=7.0 \mathrm{~Hz}, 1 \mathrm{H}), 1.98(\mathrm{~s}, 3 \mathrm{H}), 1.49(\mathrm{~d}, J=7.0 \mathrm{~Hz}, 3 \mathrm{H}) .{ }^{13} \mathrm{C} \mathrm{NMR}(75 \mathrm{MHz}$, $\left.\mathrm{CDCl}_{3}\right) \delta 169.1,143.2,128.7,127.4,126.2,48.8,23.5,21.7$. ESI-MS: $\mathrm{m} / \mathrm{z} 164[\mathrm{M}+\mathrm{H}]^{+}$

$\mathrm{N}$-benzhydrylacetamide (21). ${ }^{[27]}$ Diphenylmethanol (colorless oil; $92 \mathrm{mg}$, $0.50 \mathrm{mmol}$ ) afforded amide $\mathbf{2 1}$ as a white solid. Purified by flash column chromatography (petrol-ethyl acetate $3: 1$ ). Yield: $83 \mathrm{mg}, 74 \% . \eta=0.35$ $\mu \mathrm{L} / \mathrm{mg}$. ${ }^{1} \mathrm{H}$ NMR $\left(300 \mathrm{MHz}, \mathrm{CDCl}_{3}\right) \delta 7.34-7.18(\mathrm{~m}, 10 \mathrm{H}), 6.36(\mathrm{br} \mathrm{s}$, $1 \mathrm{H}), 6.22(\mathrm{~d}, J=8.1 \mathrm{~Hz}, 1 \mathrm{H}), 1.99(\mathrm{~s}, 3 \mathrm{H}) .{ }^{13} \mathrm{C}$ NMR $\left(75 \mathrm{MHz}, \mathrm{CDCl}_{3}\right) \delta$ 169.3, 141.6, 128.6, 127.4, 57.0, 23.2. ESI-MS: $\mathrm{m} / \mathrm{z} 226[\mathrm{M}+\mathrm{H}]^{+}, 248$ $[\mathrm{M}+\mathrm{Na}]^{+}$.

\section{Acknowledgements}

We acknowledge Croatian Ministry of Science, Education and Sports and Ruđer Bošković Institute for financial support. We are grateful to $\mathrm{Dr}$ Josip Bronić for providing the ball mill, and to Dr Ivan Halasz and Dr Krunoslav Užarević for useful discussions.
Keywords: Mechanochemistry $\bullet$ Ritter reaction $•$ Amides $•$ Organocatalysis $\cdot$ Mild conditions

[1] a) J. J. Ritter, P. P. Minieri, J. Am. Chem. Soc. 1948, 70, 4045-4048; b) J. J. Ritter, J. Kalish, J. Am. Chem. Soc. 1948, 70, 4048-4050.

[2] a) J. Clayden, N. Greeves, S. Warren, P. Wothers, Organic Chemistry, 2001; b) R. Vardanyan, V. Hruby, Synthesis of Essential Drugs, Elsevier, Amsterdam, 2006.

[3] a) L. Kurti, B. Czako, Strategic Applications of Named Reactions in Organic Synthesis, Elsevier Academic Press, Burlington, 2005; b) A Chandra, J. N. Johnston, Angew. Chem. Int. Ed. 2011, 50, 7641-7644; c) D. Stoermer, C. H. Heathcock, J. Org. Chem. 1993, 58, 564-568; d) O. L. Epstein, T. Rovis, J. Am. Chem. Soc. 2006, 128, 16480-16481; e) B. Sarmah, G. Baishya, R. K. Baruah, RSC Adv. 2014, 4, 22387-22397; f) J. S. Yadav, Y. J. Reddy, P. A. N. Reddy, B. V. S. Reddy, Org. Lett. 2013, 15, 546-549; g) B. V. S. Reddy, K. Ramesh, a. V. Ganesh, G. G. K. S. N. Kumar, J. S. Yadav, R. Grée, Tetrahedron Lett. 2011, 52, 495498.

[4] a) A. Guérinot, S. Reymond, J. Cossy, Eur. J. Org. Chem. 2012, 19-28; b) D. Jiang, T. He, L. Ma, Z. Wang, RSC Adv. 2014, 4, 64936-64946; c) F. Zhou, M. Ding, J. Zhou, Org. Biomol. Chem. 2012, 10, 3178-3181. [5] a) B. Das, K. Laxminarayana, P. Thirupathi, B. Ramarao, Synlett 2007, 3103-3106; b) M. Shi, G.-Q. Tian, Tetrahedron Lett. 2006, 47, 80598062; c) P. Rubenbauer, T. Bach, Chem. Commun. 2009, 2130-2132; d) R. Sanz, A. Martínez, V. Guilarte, J. M. Álvarez-Gutiérrez, F. Rodríguez, Eur. J. Org. Chem. 2007, 4642-4645; e) M. Barbero, S. Bazzi, S. Cadamuro, S. Dughera, Eur. J. Org. Chem. 2009, 430-436; f) M.-S. Shakeri, H. Tajik, K. Niknam, J. Chem. Sci 2012, 124, 1025 1032;

[6] a) H. Firouzabadi, A. Sardarian, H. Badparva, Synth. Commun. 1994 24, 601-607; b) S. Khaksar, E. Fattahi, E. Fattahi, Tetrahedron Lett 2011, 52, 5943-5946; c) P. Theerthagiri, A. Lalitha, P. N. Arunachalam, Tetrahedron Lett. 2010, 51, 2813-2819.

[7] a) E. Callens, A. J. Burton, A. G. M. Barrett, Tetrahedron Lett. 2006, 47, 8699-8701; b) H. Basavaprabhu, V. V Sureshbabu, Org. Biomol. Chem. 2012, 10, 2528-2533; c). Anxionnat, A. Guérinot, S. Reymond, J. Cossy, Tetrahedron Lett. 2009, 50, 3470-3473; d) G.-R. Qu, Y.-W. Song, H.-Y. Niu, H.-M. Guo, J. S. Fossey, RSC Adv. 2012, 2, 6161 6163; e) S. Yaragorla, G. Singh, P. Lal Saini, M. K. Reddy, Tetrahedron Lett. 2014, 55, 4657-4660.

[8] J. C. Baum, J. E. Milne, J. A. Murry, O. R. Thiel, J. Org. Chem. 2009 74, 2207-2209.

[9] a) M. B. Gawande, A. K. Rathi, I. D. Nogueira, R. S. Varma, P. S Branco, Green Chem. 2013, 15, 1895-1899; b) L. Ma'mani, A. Heydari, M. Sheykhan, Appl. Catal. A Gen. 2010, 384, 122-127.

[10] R. G. Kalkhambkar, S. N. Waters, K. K. Laali, Tetrahedron Lett. 2011, 52, 867-871.

[11] G. Mohammadi Ziarani, A. Badiei, Z. Dashtianeh, P. Gholamzadeh, N Mohtasham, Res. Chem. Intermed. 2013, 39, 3157-3163.

[12] N. Ibrahim, a. S. K. Hashmi, F. Rominger, Adv. Synth. Catal. 2011, 353 461-468.

[13] E. Bellamy, O. Bayh, C. Hoarau, F. Trécourt, G. Quéguiner, F. Marsais Chem. Commun. 2010, 46, 7043-7045.

[14] H. Jiang, B. Liu, Y. Li, A. Wang, H. Huang, Org. Lett. 2011, 13, 1028 31.

[15] Z. Xia, Q. Zhu, Org. Lett. 2013, 15, 4110-4113.

[16] S. L. James, C. J. Adams, C. Bolm, D. Braga, P. Collier, T. Friščić, F Grepioni, K. D. M. Harris, G. Hyett, W. Jones, et al., Chem. Soc. Rev. 2012, 41, 413-447.

[17] a) E. Boldyreva, Chem. Soc. Rev. 2013, 42, 7719-7738; b) I. Huskić, I. Halasz, T. Friščić, H. Vančik, Green Chem. 2012, 14, 1597-1600. c) G.W. Wang, Chem. Soc. Rev. 2013, 42, 7668-7700; d) K. Užarević, I. Halasz, I. Đilović, N. Bregović, M. Rubčić, D. Matković-Čalogović, V. Tomišić, Angew. Chemie Int. Ed. 2013, 52, 5504-5508. e) A. Stolle, T. Szuppa, S. E. S. Leonhardt, B. Ondruschka, Chem. Soc. Rev. 2011, 40, 
2317-2329. f) D. Gracin, V. Štrukil, T. Friščić, I. Halasz, K. Užarević, Angew. Chemie Int. Ed. 2014, 53, 6193-6197.

[18] a) K. S. McKissic, J. T. Caruso, R. G. Blair, J. Mack, Green Chem. 2014, 16, 1628-1632. b) L. Takacs, J. S. McHenry, J. Mater. Sci. 2006, $41,5246-5249$.

[19] In all experiments, tert-butanol was a liquid at ambient temperature.

[20] G. C. Gullickson, D. E. Lewis, Synthesis 2003, 681-684.

[21] The experiment with two tungsten carbide balls was not conducted. When we performed such experiments previously, balls were destroyed during milling.

[22] a) D. Braga, L. Maini, F. Grepioni, Chem. Soc. Rev. 2013, 42, 76387648; b) T. Friščić, W. Jones, Cryst. Growth Des. 2009, 9, 1621-1637.

[23] $\eta$ value is a parameter describing amount of solvent additive in respect to reagents. It is calculated as $\mathrm{V}($ solvent, $\mu \mathrm{L}) / \mathrm{m}($ sample, $\mathrm{mg})$. For details, see: T. Friščić, S. L. Childs, S. A. A. Rizvi, W. Jones, CrystEngComm 2009, 11, 418-426.

[24] Olefins are formed by the elimination of either benzyl or methyl hydrogen, and can also exist as a mixture of both elimination products. Olefin(s) were detected by TLC and were not isolated.

[25] Data corresponds to the commercially available compound

[26] E. Bellamy, O. Bayh, C. Hoarau, F. Trécourt, G. Quéguiner, F. Marsais, Chem. Commun. 2010, 46, 7043-7045.

[27] S. M. Walter, F. Kniep, E. Herdtweck, S. M. Huber, Angew. Chem. Int. Ed. 2011, 50, 7187-7191.

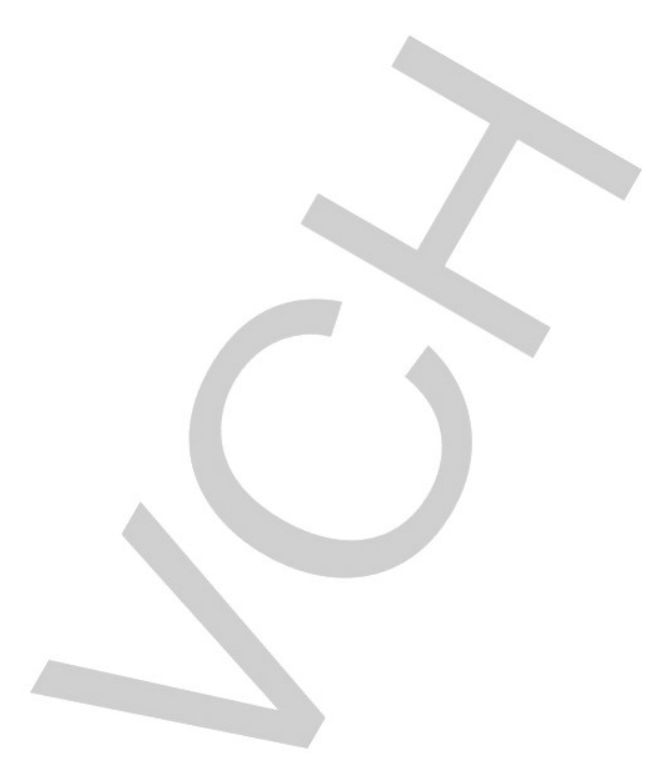




\section{FULL PAPER}

$R^{1}-\mathrm{CN}+R^{3}$
$\begin{gathered}R^{2} \\ \text { aromatic or }\end{gathered}$
$\begin{gathered}\text { tertiary or } \\ \text { aliphatic nitrile } \\ \text { alcohdary } \\ \text { ball mill, } 30 \mathrm{~min}, \mathrm{rt}\end{gathered}$

A fast and efficient mechanochemical Ritter reaction under mild conditions is described. The reaction proceeds rapidly at room temperature in a solvent-free or low-solvent environment, utilizing sulfuric acid as catalyst.
Irena Dokli, Matija Gredičak*

Page No. - Page No.

Mechanochemical Ritter reaction: a rapid approach to functionalized amides at room temperature 